\title{
Structure and biological properties of lentztrehalose: a novel trehalose analog
}

\author{
Shun-ichi Wada, Shun-ichi Ohba, Tetsuya Someno, Masaki Hatano and Akio Nomoto
}

A new trehalose analog, lentztrehalose [4-0-(2,3-dihydroxy-3-methylbutyl)trehalose], was isolated from an actinomycete Lentzea sp. Lentztrehalose is only weakly hydrolyzed by the trehalose-hydrolyzing enzyme, trehalase, so can be regarded as an enzyme-stable analog of trehalose. Although lentztrehalose does not show apparent toxicity to mammalian cells and microbes, it has antitumor activity in mice bearing S-180 sarcoma and Ehrlich carcinoma cells. In ovariectomized mice, lentztrehalose displayed a bone reinforcement effect in the femur that was superior to trehalose and induced non-morbid suppression of weight gain comparable with trehalose. These results indicate that enzyme-stable analogs of trehalose, such as lentztrehalose, may be more beneficial for human health and thus have potential as substitutes for trehalose as a sweetener.

The Journal of Antibiotics (2014) 67, 319-322; doi:10.1038/ja.2013.143; published online 22 January 2014

Keywords: antitumor; Lentzea; lentztrehalose; trehalase; trehalose

\section{INTRODUCTION}

Trehalose, a disaccharide formed from two molecules of glucose linked by an $\alpha, \alpha-1,1$-glucoside bond, is a component of tissues in animals, plants and microorganisms. In many insects, trehalose is the main sugar present in the blood. Trehalose is used as an energy source because hydrolysis of trehalose by the catabolic enzyme, trehalase, produces glucose. Although mammals do not synthesize trehalose, trehalase is expressed in the kidneys and intestine for digestion of trehalose consumed in food. ${ }^{1}$ Trehalose is used as an additive in many foods, drugs and cosmetics ${ }^{2}$ as it has a high water retention capacity. Medically relevant biological properties of trehalose include suppression of bone loss, ${ }^{3}$ antitumor effects ${ }^{4}$ and mitigation of insulin resistance. ${ }^{5}$ The wide utility of trehalose means that investigating analogs with potentially improved properties deserves exploration.

During our regular screening for antitumor agents from soil microbes, an actinomycete strain Lentzea sp. ML457-mF8 was discovered, which produced pyrazomycins and staurosporine. Although no new pyrazomycin or staurosporine compounds were found, a new trehalose analog was isolated from this strain. This compound, 4-O-(2,3-dihydroxy-3-methylbutyl)trehalose, we named lentztrehalose.

\section{RESULTS AND DISCUSSION}

Isolation and structure determination of lentztrehalose An actinomycete strain Lenzea sp. ML457-mF8 was found to produce pyrazomycins and staurosporine. To search further for new analogs and other metabolites from this strain, the culture extract was initially separated by HPLC using an octadecyl silica column. Some fractions with pyrazomycin activity were identified that contained a novel compound with a MW of 444 . This compound was then isolated by Sephadex LH-20 gel filtration. No further contamination of pyrazomycins or staurosporine was detected by TLC or HPLC. Approximately $1.0 \mathrm{~g}$ of the compound was obtained from $1 \mathrm{~kg}$ of wheat cultures of the actinomycete strain.

The actinomycete strain was isolated from a soil sample collected in Sado, Niigata Prefecture, Japan. The substrate hyphae are branched and have a pale to dull yellow color. The aerial mycelia are straight or flexuous, with fragmentation into ellipsoidal spores $(0.4-0.5 \mu \mathrm{m}$ by $1.1-1.5 \mu \mathrm{m})$. The spore surface is smooth and white. Whole-cell hydrolysates contained meso-A2pm. The partial $16 \mathrm{~S}$ ribosomal RNA (rRNA) gene sequence (1474 bp, GeneBank/EMBL/DDBJ accession number AB838595) of the strain showed high homology with those of the genus Lentzea such as L. waywayandensis (JCM $9114^{\mathrm{T}}$, T: Type strain, $1456 / 1469 \mathrm{bp}, 99.1 \%$ ) and L. albida (IFO $16102^{\mathrm{T}}$, $1458 / 1472 \mathrm{bp}, 99.0 \%)$. On the basis of taxonomic properties and the $16 \mathrm{~S}$ rRNA sequence, the strain ML457-mF8 was determined to belong to the genus Lentzea. Therefore, the strain was designated as Lentzea sp. ML457-mF8. As the new compound is a trehalose derivative from Lentzea, we coined the name lentztrehalose. No other analogs of lentztrehalose from this strain have been found so far.

The structure of lentztrehalose is shown in Figure 1a and the physicochemical properties of the compound are summarized in Table 1. The molecular formula was determined to be $\mathrm{C}_{17} \mathrm{H}_{32} \mathrm{O}_{13}$ by HR ESI-MS, elemental analysis, and NMR data (Supplementary Figures 1-5). Analysis of the ${ }^{1} \mathrm{H}-{ }^{1} \mathrm{H}$ COSY spectrum revealed two sets of spin systems (from $1^{\prime}-\mathrm{H}$ to $6^{\prime}-\mathrm{H}_{2}$ and $1^{\prime \prime}-\mathrm{H}$ to $6^{\prime \prime}-\mathrm{H}_{2}$ ), indicating the presence of two hexose moieties. The coupling constants (Table 2) indicated that the sugars are glucopyranoses. 


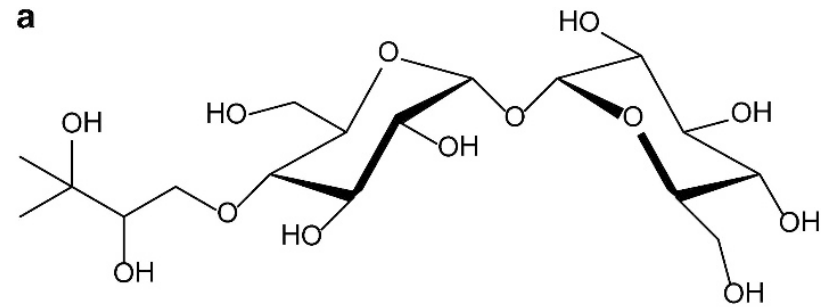

b

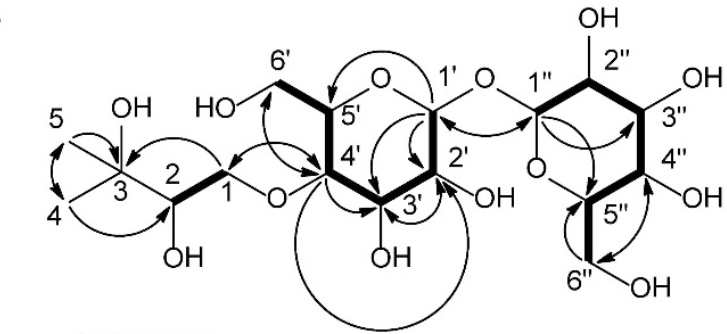

${ }^{1} \mathrm{H}-{ }^{1} \mathrm{H}$ COSY

$\frown \mathrm{HMBC}$

Figure 1 (a) Structure of lentztrehalose. (b) ${ }^{1} \mathrm{H}-{ }^{1} \mathrm{H}$ COSY and $\mathrm{HMBC}$ correlations of lentztrehalose.

Table 1 Physicochemical properties of lentztrehalose

\begin{tabular}{|c|c|}
\hline Appearance & White solid \\
\hline Optical rotation & {$[\alpha]_{D}^{22}+137(c 0.94, \mathrm{MeOH})$} \\
\hline UV $\lambda_{\max }$ & End absorption \\
\hline $\mathrm{IR}(\mathrm{KBr}) v_{\max }\left(\mathrm{cm}^{-1}\right)$ & $\begin{array}{l}3400,2935,1653,1375,1150,1079,1047 \\
991,802,610\end{array}$ \\
\hline Molecular formula & $\mathrm{C}_{17} \mathrm{H}_{32} \mathrm{O}_{13}$ \\
\hline \multicolumn{2}{|l|}{ HR ESI-MS $(\mathrm{m} / \mathrm{z})$} \\
\hline $\begin{array}{l}\text { Calculated for } \\
\mathrm{C}_{17} \mathrm{H}_{32} \mathrm{O}_{13} \mathrm{Na}\end{array}$ & 467.1735 \\
\hline Found & $467.1739(\mathrm{M}+\mathrm{Na})^{+}$ \\
\hline \multicolumn{2}{|l|}{ Elemental analysis } \\
\hline $\begin{array}{l}\text { Calculated for } \\
\mathrm{C}_{17} \mathrm{H}_{32} \mathrm{O}_{13} \cdot 2 \mathrm{H}_{2} \mathrm{O}\end{array}$ & C $42.5 \%$ H $7.5 \%$ \\
\hline Found & C $42.5 \%$ H $7.7 \%$ \\
\hline Sweetness score ${ }^{a}$ & $5.9 \pm 3.8$ (water 0, sucrose 10, trehalose $6.8 \pm 3.2)$ \\
\hline
\end{tabular}

aArbitrary score measured by a sensory test.

Ten panelists tasted $10 \mu \mathrm{l}$ of $500 \mathrm{mg} \mathrm{ml}^{-1}$ blinded solution of lentztrehalose, sucrose, trehalose and water. The sweetness of lentztrehalose and trehalose was scored on a scale where water and sucrose were scored as 0 and 10 , respectively. Mean \pm s.d.

The presence of a 2,3-dihydroxy-3-methylbutoxy moiety was established from the HMBC spectrum (Figure 1b). In the HMBC spectrum, cross-peaks were observed from the methylene protons $(\mathrm{H}-1)$ to an oxymethine carbon $\left(\mathrm{C}-4^{\prime}, \delta_{\mathrm{C}} 80.4\right)$. An HMBC correlation was also observed between $\mathrm{H}-4^{\prime}$ and a methylene carbon $(\mathrm{C}-1, \delta \mathrm{c}$ 74.7), indicating the linkage of $\mathrm{C}-1$ in the 2,3-dihydroxy-3-methylbutoxy group moiety and $\mathrm{C}-4^{\prime}$ through an oxygen atom. The configuration of the glucose moiety was determined by the mutarotase-glucose oxidase method. ${ }^{6}$ After acid hydrolysis of the compound, the reaction mixture formed a red pigment, indicating the presence of D-glucose. The optical rotation values of the compound were also consistent with $\mathrm{D}-(+)$-glucose. The presence of a 1,1-glycosidic linkage was established from the HMBC correlation between $\mathrm{C}-1^{\prime}$ and $\mathrm{C}-1^{\prime \prime}$ as shown in Figure 1b. The coupling constants
Table $2{ }^{1} \mathrm{H}$ and ${ }^{13} \mathrm{C}$ NMR data for lentztrehalose in methanol- $\mathrm{d}_{4}$

\begin{tabular}{lll}
\hline Position & \multicolumn{1}{c}{$\delta_{C^{\mathrm{a}}}$} & $\delta_{H^{\mathrm{b}}}$ \\
\hline 1 & $74.7(\mathrm{t})$ & $4.00(1 \mathrm{H}, \mathrm{dd}, J=10.6,2.8 \mathrm{~Hz}) 3.65(1 \mathrm{H}, \mathrm{m})$ \\
2 & $78.2(\mathrm{~d})$ & $3.54(1 \mathrm{H}, \mathrm{dd}, J=8.0,2.8 \mathrm{~Hz})$ \\
3 & $72.8(\mathrm{~s})$ & \\
4 & $26.4(\mathrm{q})$ & $1.18(3 \mathrm{H}, \mathrm{s})$ \\
5 & $25.4(\mathrm{q})$ & $1.16(3 \mathrm{H}, \mathrm{s})$ \\
$1^{\prime}$ & $95.1(\mathrm{~d})$ & $5.10(1 \mathrm{H}, \mathrm{d}, J=3.8 \mathrm{~Hz})$ \\
$2^{\prime}$ & $73.3(\mathrm{~d})$ & $3.50(1 \mathrm{H}, \mathrm{dd}, J=9.6,3.8 \mathrm{~Hz})$ \\
$3^{\prime}$ & $74.4(\mathrm{~d})$ & $3.90(1 \mathrm{H}, \mathrm{t}, J=9.6 \mathrm{~Hz})$ \\
$4^{\prime}$ & $80.4(\mathrm{~d})$ & $3.28(1 \mathrm{H}, \mathrm{t}, J=9.6 \mathrm{~Hz})$ \\
$5^{\prime}$ & $72.7(\mathrm{~d})$ & $3.85(1 \mathrm{H}, \mathrm{br} \mathrm{dt}, J=9.6,3.3 \mathrm{~Hz})$ \\
$6^{\prime}$ & $62.1(\mathrm{t})$ & $3.78(1 \mathrm{H}, \mathrm{m}), 3.75(1 \mathrm{H}, \mathrm{m})$ \\
$1^{\prime \prime}$ & $95.0(\mathrm{~d})$ & $5.09(1 \mathrm{H}, \mathrm{d}, J=3.8 \mathrm{~Hz})$ \\
$2^{\prime \prime}$ & $73.2(\mathrm{~d})$ & $3.45(1 \mathrm{H}, \mathrm{dd}, J=9.6,3.8 \mathrm{~Hz})$ \\
$3^{\prime \prime}$ & $74.5(\mathrm{~d})$ & $3.77(1 \mathrm{H}, \mathrm{m})$ \\
$4^{\prime \prime}$ & $71.9(\mathrm{~d})$ & $3.31(1 \mathrm{H}, \mathrm{t}, J=9.5 \mathrm{~Hz})$ \\
$5^{\prime \prime}$ & $73.8(\mathrm{~d})$ & $3.79(1 \mathrm{H}, \mathrm{m})$ \\
$6^{\prime \prime}$ & $62.6(\mathrm{t})$ & $3.78(1 \mathrm{H}, \mathrm{m}), 3.67(1 \mathrm{H}, \mathrm{dd}, J=12.0,4.7 \mathrm{~Hz})$ \\
& &
\end{tabular}

aRecorded at $150 \mathrm{MHz}$

becorded at $600 \mathrm{MHz}$.

between $\mathrm{H}-1^{\prime}$ and $\mathrm{H}-2^{\prime \prime}(J=3.9 \mathrm{~Hz})$ and between $\mathrm{H}-\mathrm{1}^{\prime \prime}$ and $\mathrm{H}-2^{\prime \prime}$ $(J=3.9 \mathrm{~Hz})$ indicated the presence of $\alpha$-glycosidic linkages.

Naturally occurring trehalose-containing molecules are relatively uncommon. To date, only 40 such compounds are registered in the database, Dictionary of Natural Products. The 2,3-dihydroxy-3methylbutoxy moiety is also rare. Among 60 compounds containing this moiety registered in the database, five are derived from fungi and the others from plants. Therefore, we believe that lentztrehalose is the first compound to contain a 2,3-dihydroxy-3-methylbutoxy moiety isolated from an actinomycete strain.

The sweetness of lentztrehalose is comparable to trehalose as shown in Table 1.

\section{Biological activity of lentztrehalose}

Lentztrehalose was not observed to have antimicrobial activity against 39 species, and 106 strains of bacteria and fungi. The MIC value was $64 \mu \mathrm{g} \mathrm{ml}^{-1}$ against 13 strains of microbes, including Haemophilus influenza and Cryptococcus neoformans and against other microbes even higher MIC values were observed. In addition, no cytotoxicity was observed with lentztrehalose at concentrations up to $200 \mu \mathrm{g} \mathrm{ml}^{-1}$ in 52 strains of human cancer cells (data not shown).

We initially considered the possibility that lentztrehalose might be a trehalase inhibitor, but lentztrehalose failed to inhibit porcine kidney trehalase up to $8 \mathrm{mg} \mathrm{ml}^{-1}$ (data not shown). However, lentztrehalose was only weakly hydrolyzed by trehalase and should be more enzyme stable than trehalose in mammals (Figure 2).

Several biological activities of trehalose in mammals have been reported. Ukawa et al. ${ }^{4}$ reported that oral administration of trehalose $25 \mathrm{mg} \mathrm{kg}^{-1}$ per day or higher markedly suppressed S-180 sarcoma tumor growth in the ICR mouse. Contrary to this report, we did not observe tumor suppression with trehalose in a similar model (Figure 3a). The antitumor effect of trehalose has been speculated to be caused by modification of the intestinal immune environment. ${ }^{4}$ Thus, the observed discrepancy in results may be due to differences in food and rearing conditions that may influence the immune system. Alternatively, the difference might be due to variations in the properties of S-180 cells because the cell line used in our experiment does not form a big tumor, such as seen with Ukawa 


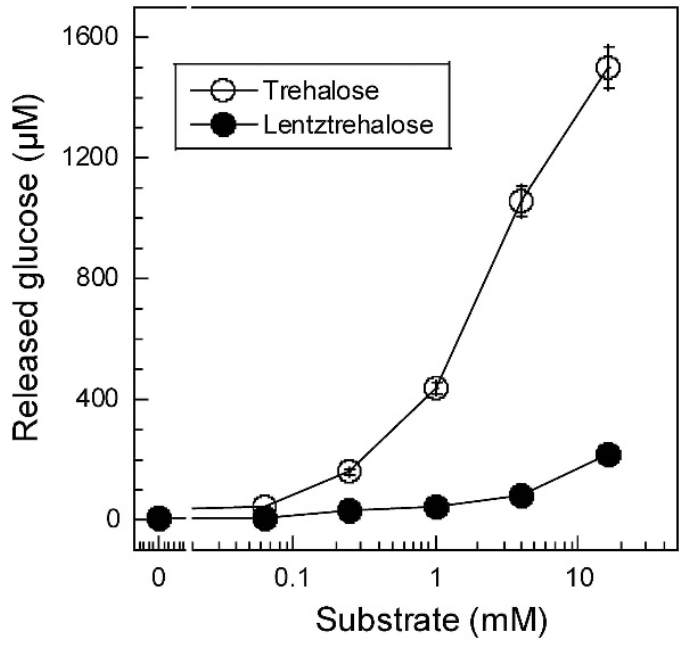

Figure 2 Hydrolysis of trehalose and lentztrehalose by porcine kidney trehalase. Trehalose or lentztrehalose was reacted with porcine kidney trehalase and the concentrations of released glucose were measured by a hexokinase assay. Mean \pm s.e. from triplicate determinations.

a

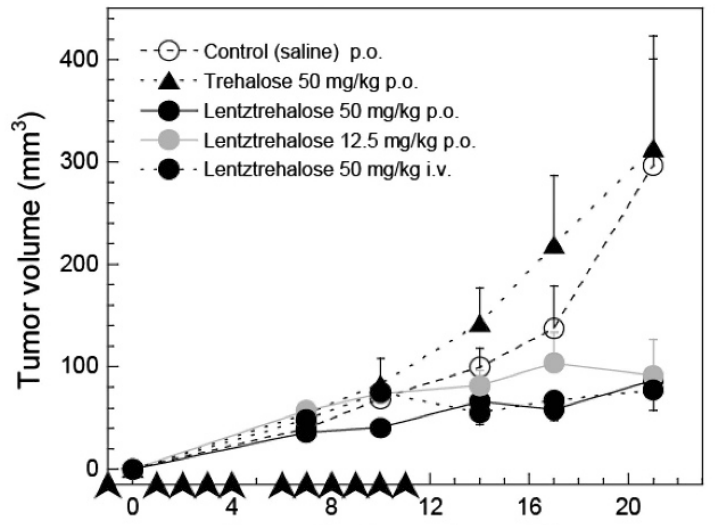

Days after tumor inoculation

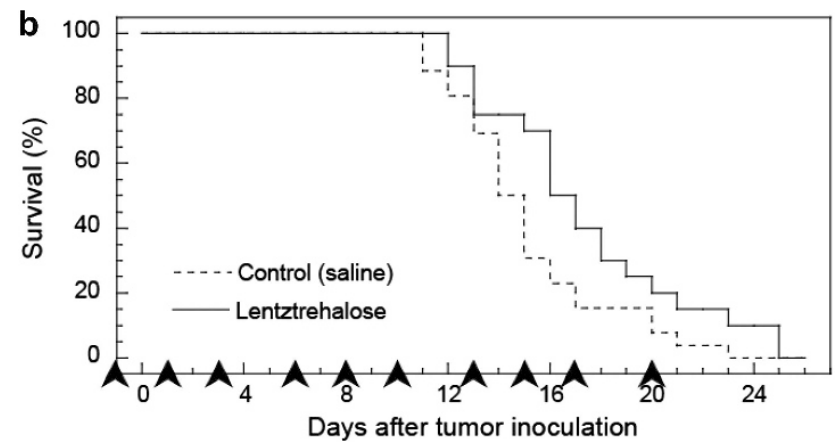

Figure 3 Antitumor effect of lentztrehalose. (a) Tumor growth in ICR mice $(n=5)$ subcutaneously inoculated with S-180 sarcoma cells. Trehalose and lentztrehalose were administrated p.o. or i.v. as indicated. Error bars indicate s.e. (b) Survival of ICR mice $(n=7)$ bearing Ehrlich ascites carcinoma cells. Lentztrehalose was administrated at $50 \mathrm{mg} \mathrm{kg}^{-1}$, p.o. Arrowheads indicate the days of sample administration.

et al. ${ }^{4}$ In our study, a higher concentration of trehalose might be required for tumor suppression. Lentztrehalose, conversely, showed potent suppression of tumor growth (Figure 3a). This effect was observed from a dose of $12.5 \mathrm{mg} \mathrm{kg}^{-1}$ per day (p.o.) and was stable at
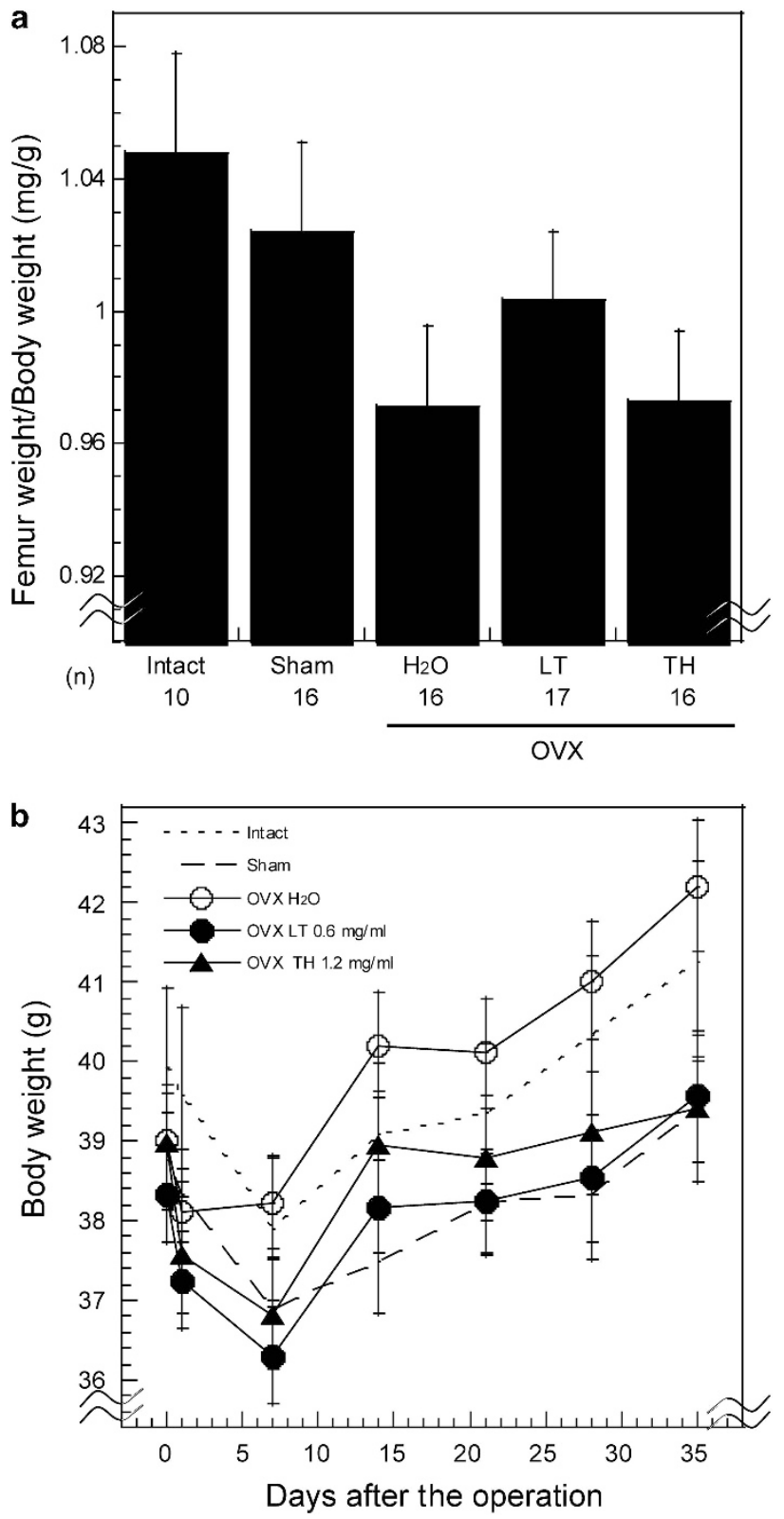

Figure 4 Bone reinforcement and anti-obesity effects of lentztrehalose in a postmenopausal mouse model. Female ICR mice (20 weeks old) were separated into intact, sham-operated and ovariectomized (OVX) groups. Intact, sham and $\mathrm{H}_{2} \mathrm{O}$ groups were given distilled water while the lentztrehalose (LT) and trehalose (TH) groups were offered water containing $0.6 \mathrm{mg} \mathrm{ml}^{-1}$ lentztrehalose and $1.2 \mathrm{mg} \mathrm{ml}^{-1}$ trehalose, respectively as the daily drink. (a) Dry weight of a femur at 5 weeks after ovariectomy. Error bars indicate s.e. (b) Body weights of mice during the experiment. Mean \pm s.e.

$50 \mathrm{mg} \mathrm{kg}^{-1}$ per day (p.o. and i.v.). We also evaluated the antitumor effect of lentztrehalose in several other mouse models. Lentztrehalose was observed to prolong the survival of ICR mice intraperitoneally harboring Ehrlich ascites carcinoma by $\sim 14.7 \%$ (Figure $3 \mathrm{~b}$ ).

The effect on bone reinforcement is one of the best studied biological activities of trehalose. ${ }^{3,7}$ We performed a similar experiment to Nishizaki et al. ${ }^{3}$ using ovariectomized mice as a postmenopausal osteoporosis model. Ovariectomized mice (Figure $4 \mathrm{a}$, OVX $\mathrm{H}_{2} \mathrm{O}$ ) lost $\sim 5 \%$ of femur weight compared with that of 
sham-operated mice. Mice provided with drinking water containing up to $1.2 \mathrm{mg} \mathrm{ml}^{-1}$ trehalose (Figure $4 \mathrm{a}$, OVX TH) did not show an obvious increase in bone weight. However, mice provided with $0.6 \mathrm{mg} \mathrm{ml}^{-1}$ lentztrehalose (OVX LT) were observed to have an increase in femur weight of $3-4 \%$. Such increases in femur weight were also observed in mice treated with $50 \mathrm{mg} \mathrm{kg}^{-1}$ lentztrehalose (p.o.) in the antitumor experiments (data not shown).

Obesity is another major problem in postmenopausal women. In ovariectomized mice, a $3.5 \%$ ( 1 week) to $7.5 \%$ (5 week) weight gain was observed compared with sham-operated controls (Figure $4 \mathrm{~b}$ ). Lentztrehalose and trehalose induced a weight reduction in ovariectomized mice to a level comparable with sham-operated mice. Toxic effects such as vomiting, diarrhea or reduction of food and drink consumption were not observed in the bone reinforcement experiment and neither compound induced weight reduction in 5 -week-old mice used in the antitumor experiment (data not shown). Therefore, the weight reduction observed with the middle-aged mouse administered lentztrehalose and trehalose was not morbid. Arai et al. ${ }^{5}$ has reported that trehalose prevents adipocyte hypertrophy. The weight loss seen with lentztrehalose is likely to be induced by the same mechanism. Therefore, lentztrehalose may also be expected to prevent obesity in middle-aged or older people if used as a diet sweetener.

The improved biological activities that we have observed with lentztrehalose compared with trehalose could be because of the higher enzyme stability of lentztrehalose. Although trehalose analogs of a similar size have been reported recently, ${ }^{8,9}$ comparative activity with trehalose was not reported. Thus, we conclude that lentztrehalose is the first potential substitute for trehalose with improved biological activity.

\section{MATERIALS AND METHODS}

Taxonomy of the actinomycete strain

The morphological properties of strain ML457-mF8 grown on ISP media No. 2, 3 and $5^{10}$ were observed with a scanning electron microscope Hitachi S-500 (Hitachi, Tokyo, Japan). For chemotaxonomic studies, cells were grown in YD medium containing $1 \%$ yeast extract and $1 \%$ glucose $(\mathrm{pH} 7.0)$ on a rotary shaker at $28^{\circ} \mathrm{C}$ for 4 days and freeze dried. Isomers of diaminopimelic acid in whole-cell hydrolysate were analyzed by the method of Staneck and Roberts. ${ }^{11}$ Analysis of $16 \mathrm{~S}$ rRNA was performed as previously described. ${ }^{12}$

\section{Isolation and structural determination of lentztrehalose}

Strain ML457-mF8 was cultured in steamed wheat at $30^{\circ} \mathrm{C}$ for 2 weeks. The whole culture was extracted with $70 \%$ ethanol and separated by sequential chromatography with several cycles of octadecyl silica HPLC (hydrosphere C18, YMC, Kyoto, Japan) and a gel filtration using Sephadex LH-20 (GE Healthcare Bio-Science AB, Uppsala, Sweden). The elution solvents were $0.1 \%$ trifluoroacetic acid for HPLC and $\mathrm{MeOH}$ for gel filtration.

The structure and physicochemical properties of lentztrehalose were determined by spectrometric analyses performed as previously described ${ }^{12}$ and using the mutarotase-glucose oxidase method. ${ }^{6}$ The novelty of lentztrehalose was confirmed with the databases Dictionary of Natural Products on DVD (Chapman \& Hall/CRC Press, Boca Raton, FL, USA) and SciFinder (Chemical Abstracts Service, Columbus, OH, USA).

\section{Trehalase assay}

Porcine kidney trehalase was purchased from Sigma-Aldrich (St Louis, MO, USA). Trehalose ( $>98 \%$ ) was purchased from Wako Pure Chemical Industries (Osaka, Japan). Lentztrehalose or trehalose was reacted with $0.24 \mathrm{uml}^{-1}$ trehalase in $135 \mathrm{~mm}$ citrate buffer $(\mathrm{pH} 7.5)$ at $37^{\circ} \mathrm{C}$ for $20 \mathrm{~min}$. The reaction was stopped by addition of an equal volume of $500 \mathrm{~mm}$ Tris- $\mathrm{HCl}$ ( $\mathrm{pH} 7.5$ ).
The released glucose was reacted with a threefold volume of Glucose Assay Reagent (Sigma-Aldrich, G3293) at room temperature for $15 \mathrm{~min}$ and the $\mathrm{A}_{340 \mathrm{~nm}}$ was measured.

\section{Antitumor assay}

The antitumor effect of lentztrehalose in ICR mice (Charles River, Yokohama, Japan) bearing S-180 sarcoma cells was evaluated according to the protocol of Ukawa et al. ${ }^{4}$ Briefly, ICR mice (5 weeks old, $q, n=5$ ) were subcutaneously inoculated with $4 \times 10^{5}$ cells per $100 \mu \mathrm{l}$ saline. Lentztrehalose (12.5-50 mg kg ${ }^{-1}$ ) and trehalose (50 mg per $\mathrm{kg}$ ) were orally or intravenously administered at days $-1,1-4$, and 6-11 after cellular inoculation. The tumor size was measured with a caliper and calculated from the formula $1 / 2$ (length $\times$ width $^{2}$ ). To evaluate the survival benefit of lentztrehalose in Ehrlich ascites carcinoma cell-bearing mice, ICR mice (5-week-old, + , $n=7$ ) were intraperitoneally inoculated with $2 \times 10^{6}$ cells per $250 \mu \mathrm{l}$ saline. Lentztrehalose $\left(50 \mathrm{mg} \mathrm{kg}^{-1}\right)$ was orally administered three times a week.

\section{Bone reinforcement assay}

The bone reinforcement effect in ovariectomized mice was examined by the modified method of Nishizaki et al. ${ }^{3}$ The ovaries of 20-week-old female ICR mice were surgically removed. The mice were kept at $23^{\circ} \mathrm{C}$ and given lentztrehalose- or trehalose-containing water as the daily drink. Intact mice were not operated upon. An abdominal incision was made in the sham group of mice and the ovaries removed once and immediately replaced at the original location followed by lesion closure.

Mice were killed at 5 weeks after the ovariectomy and both hind legs were removed. Muscle was scraped away from the femurs as much as possible with surgical scissors. The femurs were then reacted with an enzyme solution containing $0.5 \mathrm{mg} \mathrm{ml}^{-1}$ collagenase Type I (Life Technologies, Carlsbad, CA, USA) and $3.3 \mathrm{mg} \mathrm{ml}^{-1}$ dispase (Life Technologies) in phosphate buffered saline ( $\mathrm{pH}$ 7.5) (DS Pharma Biomedical, Tokyo, Japan) at $37^{\circ} \mathrm{C}$ for $10 \mathrm{~h}$. The femurs were washed with water and acetone, and weighed after desiccation.

\section{ACKNOWLEDGEMENTS}

We appreciate the help of Prof Sachi SriKantha, Gifu University, for his critical reading of this manuscript. We acknowledge the assistance of Dr M Igarashi, Dr R Sawa, Ms Y Kubota, Ms H Soutome, Mr K Inoue, Ms C Hayashi, Dr Y Takahashi and Dr K Yamazaki at the Institute of Microbial Chemistry for their support for this study and helpful discussion. This study was partially supported by the Japan Society for the Promotion of Science.

1 Elbein, A. D., Pan, Y. T., Pastuszak, I \& Carroll, D. New insights on trehalose: a multifunctional molecule. Glycobiology 13, 17R-27R (2003).

2 Ohtake, S. \& Wang, Y. J. Trehalose: current use and future applications. J. Pharm. Sci. 100, 2020-2053 (2011).

3 Nishizaki, Y. et al. Disaccharide-trehalose inhibits bone resorption in ovariectomized mice. Nutr. Res. 20, 653-664 (2000).

4 Ukawa, Y., Gu, Y., Ohtsuki, M., Suzuki, I. \& Hisamatsu, M. Antitumor effect of trehalose on sarcoma 180 in ICR mice. J. Appl. Glycosci. 52, 367-368 (2005).

5 Arai, C. et al. Trehalose prevents adipocyte hypertrophy and mitigates insulin resistance. Nutr. Res. 30, 840-848 (2010)

6 Okuda, J., Miwa, I., Maeda, K. \& Tokui, K. Rapid and sensitive, colorimetric determination of the anomers of D-glucose with D-glucose oxidase, peroxidase, and mutarotase. Carbohydr. Res. 58, 267-270 (1977).

7 Arai, C. et al. Trehalose suppresses lipopolysaccharide-induced osteoclastogenesis bone marrow in mice. Nutr. Res. 21, 993-999 (2001)

8 Kawaguchi, T. et al. Unusual amino acid derivatives from the mushroom Pleurocybella porrigens. Tetrahedron 66, 504-507 (2010).

9 Nakashima, T. et al. Trehangelins A, B and C, novel photo-oxidative hemolysis inhibitors produced by an endophytic actinomycete, Polymorphospora rubra K07-0510. J. Antibiot. 66, 311-317 (2013).

10 Shirling, E. B. \& Gottlieb, D. Methods for characterization of Streptomyces species. Int J. Syst. Bacteriol. 16, 313-340 (1966).

11 Staneck, J. L. \& Roberts, G. D. Simplified approach to identification of aerobic actinomycetes by thin-layer chromatography. Appl. Microbiol. 28, 226-231 (1974).

12 Igarashi, M. et al. Waldiomycin, a novel WalK-histidine kinase inhibitor from Streptomyces sp. MK844-mF10. J. Antibiot. 66, 459-464 (2013).

Supplementary Information accompanies the paper on The Journal of Antibiotics website (http://www.nature.com/ja) 